\title{
Increased incidence of Clostridium difficile PCR ribotype 027 in Hesse, Germany, 2011 to 2013
}

M Arvand (mardjan.arvand@hlpug.hessen.de) ${ }^{1}$, D Vollandt ${ }^{1}$, G Bettge-Weller ${ }^{1}$, C Harmanus ${ }^{2}$, E J Kuijper ${ }^{2}$, the Clostridium difficile study group Hesse ${ }^{3}$

1. Hesse State Health Office, Centre for Health Protection, Dillenburg, Germany

2. Department of Medical Microbiology, Leiden University Medical Center, Leiden, the Netherlands

3. The members of the group are listed at the end of the article

Arvand M, Vollandt D, Bettge-Weller G, Harmanus C, Kuijper El, the Clostridium difficile study group Hesse. Increased incidence of Clostridium difficile PCR ribotype 027 in Hesse, Germany, 2011 to 2013. Euro Surveill. 2014;19(10):pii=20732. Available online: http://www.eurosurveillance.org/ViewArticle.aspx?Articleld=20732

After the first outbreak of Clostridium difficile PCR ribotype (RT) 027 in Germany in 2007, no further outbreaks were reported until the recent re-emergence of RT 027 in Hesse, a federal state with 6 million inhabitants located in south-west Germany. We undertook a survey to determine the prevalence of RT 027 and other strains in a prospective study. From January 2011 to July 2013, we analysed 291 specimens from patients diagnosed with $C$. difficile infection (CDI) in 40 healthcare facilities in Hesse. The mean incidence of CDI in hospitals including at least 10 patients in the survey was 9.9 per 10,000 patient days (range $4.8-22.8$ ) in November 2012. We obtained 214 toxigenic $C$. difficile isolates. RT 001 was the most prevalent (31.8\%). RT 027 , the second most common type (26.6\%), was prevalent in all hospitals $(n=14)$ from which at least seven isolates were available for typing, but its frequency varied considerably (range: 9.1-70\%). The annual frequency of RT 027 increased from $21.4 \%$ in 2011 to $30.0 \%$ in $2013(p=0.04)$. Our study indicates that infections with $C$. difficile RT 027 are now prevalent in Hesse. It underscores the need for surveillance programmes to analyse the molecular epidemiology of C. difficile.

\section{Introduction}

Clostridium difficile is the main cause of antibiotic-associated diarrhoea in hospitals in industrialised countries. It characteristically occurs in elderly patients with co-morbidity in whom the intestinal flora has been disrupted by previous use of antibiotics. Since early 2003 , increasing rates of $C$. difficile infection (CDI) have been reported in Canada and the United States, with a larger proportion of severe and recurrent cases than previously reported $[1,2]$. The raised incidence and virulence of CDI have coincided with the spread of hypervirulent strains, particularly the NAP1/PCR-ribotype (RT) 027 strain [3]. Subsequently, epidemics of CDI caused by RT 027 have been recognised in hospitals in European countries, e.g. the United Kingdom, the Netherlands, Belgium, and Austria $[4,5]$.
In Germany, the first outbreak of infection caused by the RT 027 strain was reported from Rhineland-Palatinate in 2007 [6]. Since then, sporadic cases of infection by RT 027 have been detected in other regions, but no further outbreak has been reported. In a study from Bavaria, south-east Germany, this strain accounted for $4.6 \%$ of $C$. difficile isolates collected in 2009 [7]. In a nationwide study by Zaiss et al., RT 027 was detected in $8 \%$ of isolates obtained from patients with severe CDI in 84 German hospitals in 2008 [8]. In a pan-European survey, RT 027 was not detected among 25 C. difficile isolates collected in German hospitals in 2008, although it accounted for $5 \%$ of isolates from different European countries [9].

Mandatory reporting of severe CDI was introduced in Germany in 2007 and a case definition was developed by the Robert Koch Institute [10]. Although incidence rates and also prevalence of severe CDI increased in Germany after 2000, an association with particular strains remained unclear, since no microbiological characterisation of the isolates accompanied the nationwide surveillance [11-13]. Although we have previously reported on severe CDI due to RT 027 in Hesse, a federal state with six million inhabitants located in south-west Germany (Figure 1), our studies were mainly focussed on severe cases of $C D I$ reported to the regional health authorities $[14,15]$. Similar to the national surveillance programme, our regional surveillance did not reveal the actual prevalence of RT 027 and other circulating types. The aim of the present study was to analyse the molecular epidemiology of a comprehensive sample of C. difficile isolates associated with any clinical manifestation of CDI. We here present the results of surveillance of CDI in over 40 hospitals and other healthcare facilities in Hesse from January 2011 through July 2013.

\section{Methods}

Hospitals, rehabilitation clinics, regional health authorities and physicians in private practices were approached to voluntarily participate in the programme ' $C$. difficile surveillance in Hesse' via internet, 


\section{FIGURE 1}

Distribution of hospitals that contributed to this study by submitting at least 10 specimens from patients with Clostridium difficile infection, Hesse, 2011 to $2013(\mathrm{n}=14)$

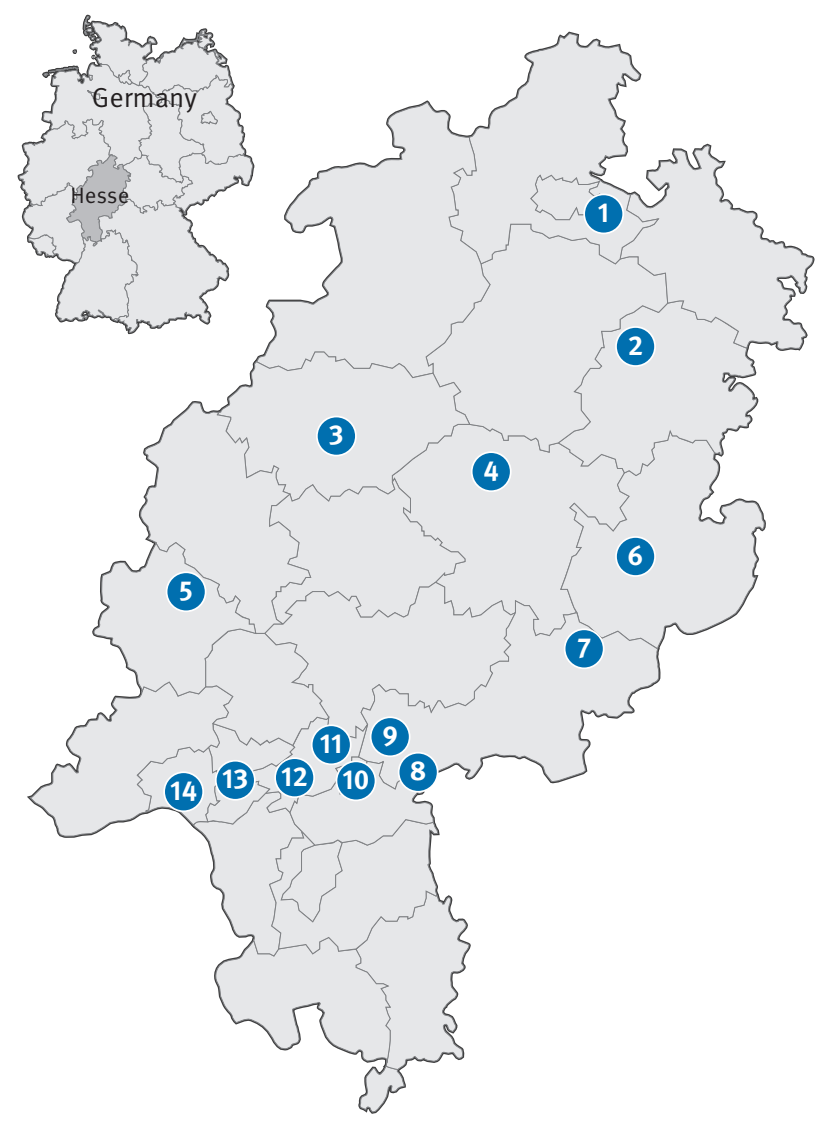

email, information events and telephone calls. Patients older than two years, who were diagnosed with CDI in a hospital or other healthcare facility in Hesse, were eligible for participation. The hospitals participating in the study were requested to enrol between 10 and 20 patients with a positive toxin test of faeces. There was no selection of patients based on specific criteria such as disease severity, duration, recurrence, etc. Patients with epidemiological link (cluster of cases or outbreak) were excluded. Patients were tested on request of their physician in the local laboratory that provided diagnostic service for the respective hospital. Participation requirements were submission of a faecal sample to our laboratories and completing a questionnaire on clinical symptoms, previous and current antibiotic therapy, and previous laboratory testing results. The participating centres also provided data to determine the incidence rates of CDI. The medical ethical committee of the Hessian Medical Association approved the investigation; no patient agreement was necessary because the samples were collected for routine microbiological diagnostics.

Additional specimens were obtained from a group of patients who had been reported to the local health authorities as severe cases of CDI (mandatory reporting). Upon request of the local health authorities to the respective hospital or local laboratory, the local diagnostic laboratory forwarded the specimens to our institution for PCR ribotyping. The isolated $C$. difficile strains $(n=12)$ were included in this study. Furthermore, some specimens from diarrhoeal patients residing in nursing homes or rehabilitation clinics were directly submitted to our laboratory for diagnostic tests for CDI. These isolates $(n=14)$ were also included in the study.

Together, 291 samples collected in 40 healthcare facilities were enrolled from January 2011 through July 2013. C. difficile was detected by culture on C. difficile-selective agar containing cycloserine, cefoxitin and fructose (Oxoid, Wesel, Germany) with and without pre-treatment with ethanol as described previously [16]. Identification was performed by routine microbiological techniques and a latex agglutination test for $C$. difficile (Microgen, Cambereley, United Kingdom). All isolates were tested for in vitro production of $C$. difficile toxins A and B by ELISA (Biopharm, Darmstadt, Germany). C. difficile toxin $A$ and $B$ genes were detected by commercial PCR kits (Hyplex, Gießen, and Hain, Nehren, Germany). PCR ribotyping was performed in the laboratories of the Hesse State Health Office or in the Department of Medical Microbiology, Leiden University Medical Center, according to the protocol of Bidet et al. [16,17].

The incidence of CDI in the collaborating hospitals was evaluated using the data provided by the study coordinator of each facility. The incidence rate was calculated by dividing the number of patients with laboratoryconfirmed CDI who were treated in the hospital in November 2012 by the total number of patient days in November 2012. This month was selected for assessing the incidence of $C D I$ in our study in order to allow a better comparison with the results of a pan-European study that analysed the incidence in the participating hospitals in November 2008 [9].

Statistical analysis was performed with the linear regression model using Excel 2007. A p value of $<0.05$ was considered significant.

\section{Results}

\section{Participating hospitals and patients' \\ characteristics}

Fourteen hospitals located in 12 cities in 11 different districts across Hesse participated in this study by submitting at least 10 faecal samples (Figure 1). Of these, 13 hospitals offered secondary or tertiary care, and one was specialised in geriatrics. Table 1 lists general information about these facilities. The samples from these hospitals accounted for 240 of 291 analysed specimens. The remaining 51 samples were obtained from 26 other healthcare facilities, including acute care hospitals, rehabilitation clinics, nursing homes, general practitioner or internist practises, and one prison. 
TABLE 1

Clostridium difficile isolates submitted by collaborating hospitals, Hesse, January 2011-July 2013 (n=180)

\begin{tabular}{|l|c|c|c|c|}
\hline Hospital number & Number of beds & $\begin{array}{c}\text { Incidence per 10,000 patient } \\
\text { days }\end{array}$ & $\begin{array}{c}\text { Number of toxigenic } \\
\text { isolates }\end{array}$ & $\begin{array}{c}\text { Number and proportion of } \\
\text { ribotype 027, } \mathrm{n}(\%)\end{array}$ \\
\hline $1^{\mathrm{a}}$ & 90 & 17.3 & 7 & $1(14.3)$ \\
\hline 2 & 570 & 4.8 & 9 & $1(11.1)$ \\
\hline 3 & 1,200 & 7.9 & 16 & $2(12.5)$ \\
\hline 4 & 190 & 5.1 & 12 & $1(9.1)$ \\
\hline 5 & 180 & 7.3 & 8 & $3(33.3)$ \\
\hline 6 & 930 & NA & 13 & $4(37.5)$ \\
\hline 7 & 280 & 11.3 & 10 & $7(70.0)$ \\
\hline $8^{\mathrm{b}}$ & 270 & 12.6 & 17 & $2(11.8)$ \\
\hline 9 & 750 & 5.4 & 14 & $2(14.3)$ \\
\hline 10 & 790 & 11.5 & 20 & 9 \\
\hline 11 & 1,190 & 5.9 & 16 & $9(45.0)$ \\
\hline 12 & 1,030 & 11.2 & 11 & $4(25.0)$ \\
\hline $13^{\mathrm{b}}$ & 140 & 22.8 & 16 & $1(9.1)$ \\
\hline 14 & 1,030 & 5.6 & & $9(56.3)$ \\
\hline
\end{tabular}

NA: not available.

a Geriatric hospital.

b Hospital with large geriatric unit.

All submitting hospitals were acute care hospitals located in 12 cities in 11 different districts in Hesse and submitted at least 10 specimens from patients with Clostridium difficile infection to this study (Figure 1).

Of 291 samples analysed, 229 samples which were collected from 219 patients contained C. difficile. From 219 patient-adjusted isolates, 214 (97.7\%) were toxigenic. Together, 193 (90.2\%) of 214 toxigenic isolates were from patients in acute care hospitals, nine (4.2\%) from rehabilitation clinics, seven (3.3\%) from outpatients, and five (2.3\%) from nursing homes. Four of seven outpatients had been hospitalised within four weeks before onset of diarrhoea. These cases were considered as hospital-acquired.

The median age of patients with confirmed CDI $(n=214)$ was 77 years (range: 2.5-98 years), and 107 patients (49.5\%) were female. According to the questionnaire, $36(16.7 \%)$ patients had previous CDI episodes in the three months before the current episode and 20 (9.3\%) patients had died at the time the questionnaire was completed, i.e. within 30 day after collection of the faecal sample.

\section{Characteristics of Clostridium difficile isolates}

The 214 toxigenic isolates were further characterised. Altogether, 41 ribotypes were detected. Ribotype 001 was the most prevalent type, accounting for 68 (31.8\%) of toxigenic isolates (Table 2). It was detected in 20 of 37 healthcare facilities that had submitted samples with positive culture results. Ribotype 027 represented the second most common strain, accounting for $57(26.6 \%)$ of toxigenic isolates. It was detected in 19 healthcare facilities. One isolate was defined as a RT 027 variant because it displayed a slight banding difference compared to the epidemic RT 027 strain. In contrast to the epidemic RT 027, this isolate was sensitive to erythromycin and moxifloxacin. But similar to the epidemic RT 027 strain, this isolate also contained binary toxin genes and had a mutation in $t c d C$ at positions 18 and 117. Ribotype 014 accounted for $21(9.8 \%)$ of toxigenic isolates and was detected in 13 healthcare facilities. Ribotypes 078, 002, 029, 012, 017 , and 005 were encountered in $2.8 \%, 2.8 \%, 2.8 \%$, $1.9 \%, 1.9 \%$ and $1.4 \%$ of toxigenic isolates (Table 2 ). Other ribotypes were detected sporadically, i.e. once or twice, in this study. They included RT 003, 011, 013, 043, 045, 046, 049, 052, 062, 071, 081, 087, 126, 136, 150, 159, 181, 207, 209, 216, 235, 258, 268, 293, 476, and seven unknown ribotypes.

\section{Prevalence of Clostridium difficile RT 027 in} the collaborating hospitals

We next determined the frequency of isolation of RT 027 in those hospitals that had submitted at least 10 specimens to this study (Figure 1). The epidemic RT 027 strain was detected in all hospitals, but its prevalence varied markedly between different hospitals, ranging from 9.1 to $70 \%$ (Table 1 ).

\section{Incidence of Clostridium difficile infection in the collaborating hospitals}

We further evaluated the incidence of CDI in collaborating hospitals that had submitted at least 10 specimens from CDI patients to this study. Incidence data were collected for one month (November 2012). Data were available from 13 of 14 hospitals. The mean incidence 
TABLE 2

Frequency of Clostridium difficile ribotypes among toxigenic isolates from 37 healthcare facilities in Hesse, January 2011 to July 2013 ( $\mathrm{n}=214$ isolates)

\begin{tabular}{|l|c|c|c|}
\hline Ribotype & $\begin{array}{c}\text { Number of } \\
\text { isolates }\end{array}$ & $\begin{array}{c}\text { Proportion of } \\
\text { isolates (\%) }\end{array}$ & $\begin{array}{c}\text { Number of } \\
\text { healthcare } \\
\text { facilities }\end{array}$ \\
\hline 001 & 68 & 31.8 & 20 \\
\hline 027 & 57 & 26.6 & 19 \\
\hline 014 & 21 & 9.8 & 13 \\
\hline 078 & 6 & 2.8 & 6 \\
\hline 002 & 6 & 2.8 & 4 \\
\hline 029 & 6 & 2.8 & 4 \\
\hline 012 & 4 & 1.9 & 3 \\
\hline 017 & 4 & 1.9 & 1 \\
\hline 005 & 3 & 1.4 & 2 \\
\hline
\end{tabular}

In total, 39 different ribotypes were detected. Ribotypes detected only once or twice in this study are not listed in the Table.

of CDI in these hospitals was 9.9 per 10,000 patient days. The incidence varied considerably between different hospitals, ranging from 4.8 to 22.8 per 10,000 patient days (Table 1). The highest incidence rates were reported from a geriatric clinic (Hospital 1: 17.3 per 10,000 patient days) and a hospital with a large geriatric unit (Hospital 13: 22.8 per 10,000 patient days).

\section{Changing frequency of Clostridium difficile RT} 027 over time

In order to monitor the spread of RT 027, we determined the annual frequency of isolation of RT 027, along with other prevalent ribotypes such as RT 001 and 014, over the study period. Ribotype 027 accounted for $21.4 \%$ of toxigenic isolates in 2011. Its frequency increased to $26.2 \%$ in 2012 and $30.0 \%$ in 2013 (Figure 2). The increase in the prevalence of RT 027 was statistically significant (correlation coefficient: 0.995; $p=0.04$ ).

\section{Discussion}

To our knowledge, this is the first report on increased incidence of $C$. difficile RT 027 in Germany. The epidemic RT 027 strain represented the second most prevalent type and accounted for $26.6 \%$ of isolates collected in 37 healthcare facilities in Hesse in 2011 to 2013. Previous studies have revealed a relatively low prevalence (0-8\%) of RT 027 among isolates collected in different parts of Germany, including Hesse, in 2008 and 2009 [7-9]. RT 027 was not detected in a random collection of $C$. difficile isolates from German hospitals in 2008 [9]. More recent data from a representative nation-wide sample are not yet available. Our data suggest that the epidemiology of RT 027 has changed in Hesse and probably also in other parts of Germany.

Changing epidemiology of $\mathrm{CDI}$ has been recently reported from other European countries $[5,18]$. A substantial increase in the incidence of CDI and prevalence of RT 027 was observed in England and Wales in 2005 to $2007[19,20]$. The introduction of a bundle of infection control measures at national level led to a significant decrease in the incidence of CDI and prevalence of RT $027[5,18]$. In the Netherlands, RT 027 was associated with outbreaks in several hospitals in 2005 and 2006 [21]. Its prevalence decreased in the period from 2006 to 2009 and seemed to remain stable thereafter [22]. However, recent reports in 2013 suggest reemergence of RT 027 associated with severe CDI and outbreaks in the Netherlands, especially in healthcare facilities associated with nursing homes [23,24].

In our study, $C$. difficile RT 027 was prevalent in all hospitals from which a sufficient number of isolates were available for typing, but its frequency varied between hospitals. Higher prevalence rates were observed in the southern part of Hesse (Hospitals 5-14), which is characterised by a higher density of population and hospitals. However, we also observed striking differences between hospitals in the same city. For example, the prevalence of RT 027 was $70 \%$ in Hospital 8 and $11.8 \%$ in Hospital 9, which are located in the same city. Similarly, Hospitals 11 and 12 were located in the same city and had a different prevalence of RT 027 ( $45 \%$ versus $25 \%$ ). Differences in patient characteristics, antimicrobial therapy regimens, diagnostic tests, awareness of the doctors, and infection prevention policy may have contributed to this variability.

None of the participating hospitals in this study had noticed or reported an outbreak of CDI at the time of sample collection. Nonetheless, the high prevalence of RT 027 in some hospitals indicates possible ongoing transmission. Further investigations to evaluate this hypothesis are in progress. Together, our data suggest that RT 027 has become endemic in hospitals in Hesse. Because RT 027 is associated with outbreaks, more severe diarrhoea, higher attributable mortality, and more recurrences than other ribotypes, our findings underscore the need for effective infection control measures to curb the spread of RT 027 and other hypervirulent strains in Germany.

The mean incidence of CDI in the collaborating hospitals was 9.9 per 10,000 patient days in our study. This is higher than the rate reported by the national surveillance system CDAD-KISS in 2012 (7.2 per 10,000 patient days) [25]. This difference may in part be due to methodological differences. Our incidence data were derived from one month (November 2012), whereas the KISS data include the whole year. It is also possible that the high prevalence of RT 027 in our region may have contributed to a higher incidence of CDI in our study. However, it is also plausible that differences in patients' characteristics and selection of participating hospitals could have contributed to this discrepancy. The participating hospitals in CDAD-KISS are mainly large academic hospitals. None of the collaborating hospitals in our study were participants of CDAD-KISS. 


\section{FIGURE 2}

Frequency of Clostridium difficile ribotypes 027, 001, 014, and other ribotypes among toxigenic C. difficile isolates from Hesse,

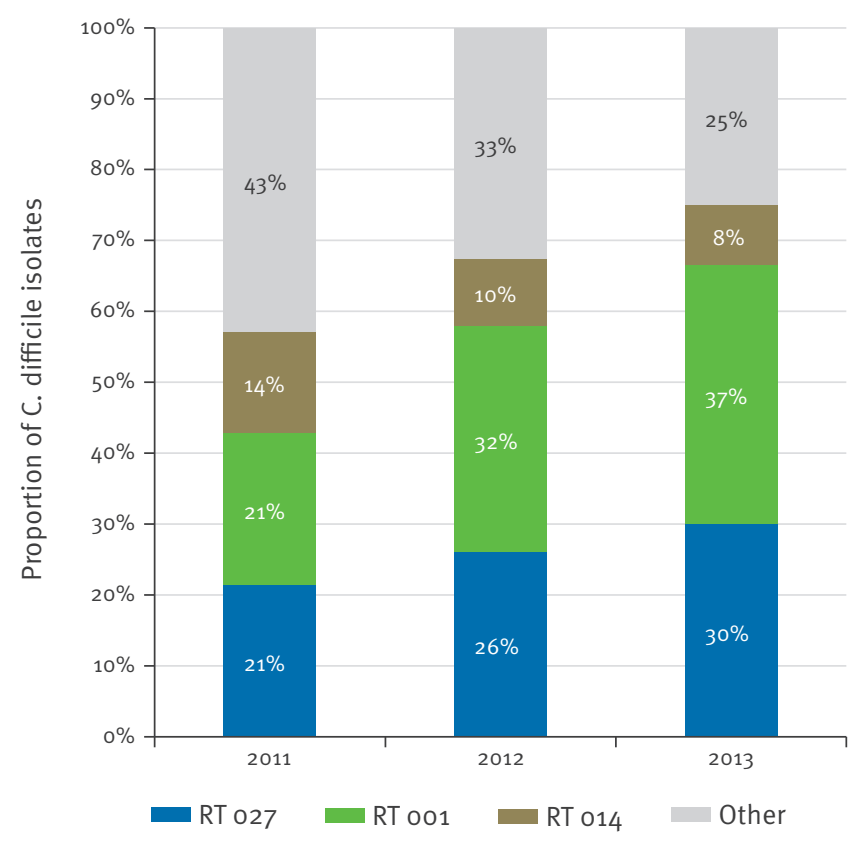

RT: ribotype

The percentage of isolates assigned to each ribotype is shown in the columns.

Note: data for 2013 include only seven months.

Our study included several smaller hospitals with a large proportion of geriatric patients, who are generally at higher risk of developing CDI. It is important to note that the highest incidence rates in our study were observed in geriatric hospitals and those with a large geriatric unit.

In a pan-European survey, the incidence of hospitalassociated CDI ranged from o to 19.1 per 10,000 patient days in different countries [9]. In comparison, the incidence of CDI in our study seems moderate. However, we believe that the incidence of CDI is generally underestimated in Germany, mainly because diagnostic tests are not routinely performed for all patients with healthcare-associated diarrhoea. Further educational programmes are necessary to increase the awareness of doctors and healthcare personnel for CDI.

The main limitation of our study is the small sample size, which can be explained by the voluntary nature of participation and limitations in time and resources in the collaborating hospitals. Since the study is being continued, we hope to overcome this restriction by increasing the number of the samples analysed.

In conclusion, $C$. difficile infections caused by RT 027 are now observed frequently in Hesse and their prevalence seems to be increasing. Our data underscore the need for surveillance programmes that include both microbiological and epidemiological data at regional, state, and national level in Germany and for intervention programmes to combat CDI and the spread of hypervirulent strains.

\section{Acknowledgments}

This project was partially supported by the German Ministry of Health (regional networks for prevention and control of antimicrobial resistance). We are grateful to all participating hospitals and healthcare facilities for submitting specimens from case patients, and to the personnel of regional health offices for cooperation with this project. We thank Bettina Scholl, Vera Martin, and Susanne Winter for excellent technical assistance, and Vanessa Ascherl for help with Figure 1.

Conflict of interest

None declared.

Authors' contributions

MA designed and conducted the survey

DV, GB-W, CH, EK, and MA performed laboratory investigation and data analysis

MA, wrote the manuscript

The C. difficile study group Hesse coordinated the study in the hospitals, enrolled patients, and collected data

Clostridium difficile study group Hesse

Dr. Sabine Albert-Braun and Dr. Jörg Schulze, Klinikum Frankfurt Höchst, Frankfurt am Main, and Sana Klinikum Offenbach,

PD Dr. Christian Brandt, Klinikum der Goethe-Universität, Frankfurt am Main,

Dr. Wolfgang Dembowski and Ankica Scheidel, St. VinzenzKrankenhaus Hanau,

Dr. Johannes-Georg Elsing, Krankenhaus des Vogelsbergkreises, Alsfeld,

Dr. Markus Hofmann, Kreiskrankenhaus Weilburg, Dr. Martina Kerwat, Universitätsklinikum Gießen und Marburg, Standort Marburg,

Dr. Andreas Kneifel, Klinikum Hanau,

Dr. Hans Milerski, Deutsches Rotes Kreuz Klinik Kaufungen,

Dr. Dr. Klaus-Peter Reetz, Kliniken des Main-Taunus-Kreises, Krankenhaus Hofheim,

Dr. Christine Schindel, Dr. Horst Schmidt Kliniken, Wiesbaden,

Rolf Suter, Herz-und Kreislaufzentrum Rotenburg an der Fulda,

Dr. Martin Wemuth, Main-Kinzig-Kliniken, Krankenhaus Schlüchtern. 


\section{References}

1. Loo VG, Poirier L, Miller MA, Oughton M, Libman MD, Michaud $\mathrm{S}$, et al. A predominantly clonal multi-institutional outbreak of Clostridium difficile-associated diarrhea with high morbidity and mortality. N Engl J Med. 2005;353(23):2442-9. http://dx.doi.org/10.1056/NEJMoa051639

2. McDonald LC, Killgore GE, Thompson A, Owens RC, Jr., Kazakova SV, Sambol SP, et al. An epidemic, toxin gene-variant strain of Clostridium difficile. N Engl J Med. 2005;353(23):243341. http://dx.doi.org/10.1056/NEJMoao51590

3. Freeman J, Bauer MP, Baines SD, Corver J, Fawley WN, Goorhuis $\mathrm{B}$, et al. The changing epidemiology of Clostridium difficile infections. Clin Microbiol Rev. 2010;23(3):529-49. http://dx.doi.org/10.1128/CMR.00082-09

4. Kuijper EJ, Coignard B, Brazier JS, Suetens C, Drudy D, Wiuff C, et al. Update of Clostridium difficile-associated disease due to PCR ribotype 027 in Europe. Euro Surveill. 2007;12(6):pii=714.

5. Jones AM, Kuijper EJ, Wilcox MH. Clostridium difficile: a European perspective. J Infect. 2013;66(2):115-28. http://dx.doi.org/10.1016/j.jinf.2012.10.019

6. Kleinkauf N, Weiss B, Jansen A, Eckmanns T, Bornhofen B, Kuehnen E, et al. Confirmed cases and report of clusters of severe infections due to Clostridium difficile PCR ribotype 027 in Germany. Euro Surveill. 2007;12(46): pii=3307.

7. Reil M, Hensgens MP, Kuijper EJ, Jakobiak T, Gruber H, Kist M, et al. Seasonality of Clostridium difficile infections in Southern Germany. Epidemiol Infect. 2012;140(10):1787-93. http://dx.doi.org/10.1017/So950268811002627

8. Zaiss NH, Witte W, Nubel U. Fluoroquinolone resistance and Clostridium difficile, Germany. Emerg Infect Dis. 2010;16(4):675-7. http://dx.doi.org/10.3201/eid1604.090859

9. Bauer MP, Notermans DW, van Benthem BH, Brazier JS, Wilcox $M H$, Rupnik M, et al. Clostridium difficile infection in Europe: a hospital-based survey. Lancet. 2011;377(9759):63-73. http://dx.doi.org/10.1016/S0140-6736(10)61266-4

10. Robert Koch Institute. Schwer verlaufende Infektionen mit Clostridium difficile: Zur Meldepflicht. [Severe infections with Clostridium difficile: on mandatory reporting]. Epidemiologisches Bulletin. 2007;46:424. German. Available from: http://www.gpk.de/downloadp/STIKO_2007 Bulletin46_071116_Zunahme_von_Norovirus_Infektionen_ koennte_erneute.pdf

11. Vonberg RP, Schwab F, Gastmeier P. Clostridium difficile in discharged inpatients, Germany. Emerg Infect Dis. 2007;13(1):179-80. http://dx.doi.org/10.3201/eid1301.060611

12. Borgmann $S$, Kist M, Jakobiak T, Reil M, Scholz E, von EichelStreiber C, et al. Increased number of Clostridium difficile infections and prevalence of Clostridium difficile PCR ribotype 001 in southern Germany. Euro Surveill. 2008;13(49):pii=19057.

13. Robert Koch Institute. Schwer verlaufende Clostridiumdifficile-Infektionen: IfSG-Surveillancedaten von 2011 und 2012. [Severe infections with Clostridium difficile: surveillance data from 2011 and 2012]. Epidemiologisches Bulletin. 2013;25:233-7. German. Available from: https://www.rki.de/ DE/Content/Infekt/EpidBull/Archiv/2013/Ausgaben/25_13. pdf?__blob=publicationFile

14. Arvand M, Hauri AM, Zaiss NH, Witte W, Bettge-Weller G. Clostridium difficile ribotypes 001,017 , and 027 are associated with lethal C. difficile infection in Hesse, Germany. Euro Surveill. 2009;14(45):pii=19403.

15. Arvand M, Hauri AM, Zaiss NH, Witte W, Bettge-Weller G. [Epidemiology of severe Clostridium difficile infections in Hesse, Germany in 2008-2009]. Dtsch Med Wochenschr. 2010;135(40):1963-7. http://dx.doi.org/10.1055/s-0030-1263342

16. Arvand M, Moser V, Schwehn C, Bettge-Weller G, Hensgens MP, Kuijper EJ. High prevalence of Clostridium difficile colonization among nursing home residents in Hesse, Germany. PLoS ONE. 2012;7(1):e30183. http://dx.doi.org/10.1371/journal.pone.0030183

17. Bidet P, Barbut F, Lalande V, Burghoffer B, Petit JC. Development of a new PCR-ribotyping method for Clostridium difficile based on ribosomal RNA gene sequencing. FEMS Microbiol Lett. 1999;175(2):261-6. http://dx.doi.org/10.1111/j.1574-6968.1999.tb13629.x

18. Wilcox MH, Shetty N, Fawley WN, Shemko M, Coen P, Birtles $A$, et al. Changing epidemiology of Clostridium difficile infection following the introduction of a national ribotyping based surveillance scheme in England. Clin Infect Dis. 2012;55(8):1056-63.

http://dx.doi.org/10.1093/cid/cis614
19. Brazier JS, Patel B, Pearson A. Distribution of Clostridium difficile PCR ribotype 027 in British hospitals. Euro Surveill. 2007;12(17): pii=3182.

20. Brazier JS, Raybould R, Patel B, Duckworth G, Pearson A, Charlett $A$, et al. Distribution and antimicrobial susceptibility patterns of Clostridium difficile PCR ribotypes in English hospitals, 2007-08. Euro Surveill. 2008;13(41):pii=19000.

21. Kuijper EJ, van den Berg RJ, Debast S, Visser CE, Veenendaal D, Troelstra A, et al. Clostridium difficile ribotype 027, toxinotype III, the Netherlands. Emerg Infect Dis. 2006;12(5):827-30. http://dx.doi.org/10.3201/eid1205.051350

22. Hensgens MP, Goorhuis A, Notermans DW, van Benthem $\mathrm{BH}$, Kuijper EJ. Decrease of hypervirulent Clostridium difficile PCR ribotype 027 in the Netherlands. Euro Surveill. 2009;14(45): $\mathrm{pii}=19402$.

23. Knetsch CW, Lawley TD, Hensgens MP, Corver J, Wilcox MW, Kuijper EJ. Current application and future perspectives of molecular typing methods to study Clostridium difficile infections. Euro Surveill. 2013;18(4): pii=20381.

24. Seventh annual report of the national reference laboratory for Clostridium difficile and results of the national surveillance, May 2012 to May 2013. Leiden: University Medical Center and Bilthoven: Center for Infectious Diseases Control; 2013. Available from: http://www.rivm.nl/dsresource?objectid=rivmp :211067\&type=org\&disposition=inline\&ns_nc=1

25. KISS Krankenhaus-Infektions-Surveillance-System. Modul CDAD-KISS. Referenzdaten Berechnungszeitraum 1. Januar 2012 bis 31. Dezember 2012. [KISS hospital infection surveillance system. Module CDAD-KISS. Reference data evaluation period 1 January 2012 to 31 December 2012]. Berlin: Nationales Referenzzentrum für Surveillance von nosokomialen Infektionen; 2013; German. Available from: http://www.nrzhygiene.de/surveillance/kiss/cdad-kiss/da62C252/995/1365/ 